\title{
Considerations on the identification and management of metastatic prostate cancer patients with DNA repair gene alterations in the Canadian context
}

\begin{abstract}
Michael P. Kolinsky ${ }^{1}$; Karen Y. Niederhoffer ${ }^{2}$; Edmond M. Kwan ${ }^{3}$; Sebastien J. Hotte ${ }^{4}$; Zineb Hamilou$^{5}$; Steven M. Yip ${ }^{6}$; Kim N. Chi ${ }^{7}$; Alexander W. Wyatt ${ }^{8}$; Fred Saad ${ }^{9}$

${ }^{1}$ Cross Cancer Institute, Edmonton, Alberta, Canada; Department of Oncology, University of Alberta, Edmonton, AB, Canada; ${ }^{2}$ Department of Medical Genetics, University of Alberta, Edmonton, AB, Canada; ${ }^{3}$ Vancouver Prostate Centre, Department of Urologic Sciences, University of British Columbia, Vancouver, BC, Canada; ${ }^{4}$ Department of Oncology, McMaster University, Hamilton, ON, Canada; ${ }^{5}$ Division of Oncology, Centre Hospitalier de l'Université de Montréal, Montreal, QC, Canada; ${ }^{6}$ Tom Baker Cancer Centre and Cumming School of Medicine, Calgary, AB, Canada; ${ }^{7}$ BC Cancer Agency and University of British Columbia, Vancouver, BC, Canada; ${ }^{8}$ Vancouver Prostate Centre and Department of Urologic Sciences, University of British Columbia and Michael Smith Genome Sciences Centre, BC Cancer, Vancouver, BC, Canada; ${ }^{9}$ Centre Hospitalier de l'Université de Montréal, Université de Montréal, Montreal, QC, Canada
\end{abstract}

Cite as: Kolinsky MP, Niederhoffer KY, Kwan EM, et al. Considerations on the identification and management of metastatic prostate cancer patients with DNA repair gene alterations in the Canadian context. Can Urol Assoc J 2021 November 18; Epub ahead of print. http://dx.doi.org/10.5489/cuaj.7621

Published online November 18, 2021

Corresponding author: Dr. Fred Saad, Centre Hospitalier de l'Université de Montréal, Université de Montréal, Montreal, QC, Canada; fredsaad@videotron.ca

$* * *$

\section{Summary}

Olaparib is the first Health Canadaapproved agent in metastatic prostate cancer to use a companion diagnostic to identify alterations in $B R C A 1, B R C A 2$, or ATM. As olaparib is introduced, clinicians must learn to access and interpret germline and somatic nextgeneration sequencing (NGS) results, and how to manage affected patients who appear to have distinct clinical features. The traditional model of referring patients to a hereditary cancer clinic

\section{Key messages:}

- With the Health Canada approval of olaparib in mCRPC, the era of molecular classification and biomarker-driven therapy in advanced prostate cancer has arrived.

- Canadian clinicians will need to be familiar with how to access and interpret germline and somatic genetic testing results.

- Clinicians can advocate for provincial reimbursement, as well as increased resources and optimal pathways for germline and somatic genetic testing, including oncology provider-mediated genetic testing ("mainstreaming").

- The field is continually evolving, and many questions remain unanswered, including optimal patient selection and sequencing of olaparib with other standard-of-care therapies. 
(HCC) for germline testing is likely impractical in this disease, as the metastatic prostate cancer patient population would be overwhelming. Alternate approaches to this are clinician-ordered genetic testing (so-called "mainstreaming"), out-of-pocket payment for third-party private company genetic testing, or germline testing done in conjunction with somatic testing, particularly cell free circulating tumor DNA (ctDNA).

Germline testing alone is not sufficient for identifying Olaparib-eligible patients, as less than half of $B R C A 1, B R C A 2$, or $A T M$ alterations are germline in origin, but it is critically important to identify family members who are carriers so that risk-reduction measures can be undertaken. Somatic testing is not widely available in Canada, but some patients can access it through research protocols or by paying out-of-pocket. Somatic testing can be performed on archival or fresh solid tissue biopsy samples, or through whole blood samples to access plasma-derived circulating tumor DNA (ctDNA). Both testing approaches have relative advantages and disadvantages, but neither may be informative in all patients and, therefore, ideal somatic NGS pathways should provide options for both tissue and ctDNA testing.

We advocate that clinicians begin discussions with their provincial lab formularies, HCC, and molecular pathology labs to highlight the importance of germline and somatic testing in this population and identify pathways for patient access. While olaparib has approval for use in $B R C A 1, B R C A 2$, and ATM-altered mCRPC, emerging evidence suggests that PARP inhibitors have variable activity in these three genes, with $B R C A 2$ alterations appearing to be the most responsive. Retrospective and prospective series have reported varying outcomes to standard of care therapies, such as ARATs and taxane-based chemotherapy, in metastatic castration-resistant prostate cancer (mCRPC) patients with DNA damage repair (DDR) gene alterations, such as $B R C A 2$. In the absence of high-level evidence showing a lack of benefit, we believe this patient population should still be considered for these treatments.

In addition, platinum-based chemotherapy appears to have activity in DDR gene-altered mCRPC and should be considered another option when access to olaparib is not possible.

At present, there is no evidence to support an optimal treatment sequence in this patient population, therefore, physician and patient preferences will need to be taken into consideration when selecting therapies. As olaparib and other PARP inhibitors are tested in different disease states and in combination with other therapies, we will likely see a more refined approach to use of these agents and management of this new biomarker-defined patient population.

\section{Introduction}

Since the pivotal discovery of Canadian-born physician, Dr. Charles Huggins, that prostate cancer is dependent on androgenic activity ${ }^{1}$ a number of life-prolonging systemic therapies have been developed and approved by Health Canada: docetaxel ${ }^{2}$, cabazitaxel $^{3}$, abiraterone ${ }^{4}$, enzalutamide $^{5}$, apalutamide ${ }^{6}$, darolutamide ${ }^{7}$, radium $223^{8}$, sipileucel $\mathrm{T}^{9}$, and olaparib ${ }^{10}$. Based on the recently published VISION study, Lutetium-177-PSMA-617 will likely also receive Health Canada approval ${ }^{11}$. 
The Health Canada approval of olaparib for patients with metastatic castration-resistant prostate cancer (mCRPC) who have progressed after at least one line of androgen receptor axis targeted therapy (ARAT), with deleterious or suspected deleterious pathogenic germline and/or somatic variants in either $B R C A 1, B R C A 2$, or $A T M$ represents a significant shift in the diagnostic and therapeutic landscape of metastatic prostate cancer (mPC). Prior to olaparib, all of the systemic therapies approved for $\mathrm{mPC}$ have been with no companion diagnostic or clinically validated biomarkers to identify patients most likely to benefit. As such, how to access and interpret molecular testing, particularly from next generation sequencing (NGS) technologies, will be foreign to many Canadian prostate cancer health care providers. This review aims to address two broad topics in the context of the Canadian Health care system: 1) testing for DNA damage repair (DDR) gene alterations in patients with $\mathrm{mPC}$, through germline and/or somatic testing; and 2) management of the MPC patient with DDR gene alterations, including the use of olaparib and other considerations for therapy.

\section{Prevalence of DDR gene alterations in advanced prostate cancer}

Genomic studies have demonstrated that approximately a quarter of mCRPC patients harbour alterations in DDR genes, with BRCA2 (13\%) and ATM (7\%) being the most frequently affected $^{12-15}$. Less than half of these alterations are germline in origin, with the remainder of patients harbouring somatic only alterations (table 1). The prevalence of germline DDR gene alterations appears to varies by population. One multicentre study demonstrated an overall germline mutation prevalence of $11.8 \%$ for any DDR gene, (or $7.8 \%$ if only considering $B R C A 1$, $B R C A 2$ or $A T M)$, though this ranged from $8.8 \%$ to $18.5 \%$ by centre ${ }^{16}$. A recent study from 879 $\mathrm{mPC}$ patients in British Columbia found a prevalence of germline DDR gene alterations of 6.5\%, and $4.9 \%$ in $B R C A 1, B R C A 2$, or $A T M^{15}$. Whether this is representative of other Canadian jurisdictions is not yet known.

\section{Testing for DDR gene alterations in advanced prostate cancer}

\section{Germline testing}

Germline testing identifies variants that are present in all cells in the body. These changes are typically inherited from a parent and can be passed on to children. Patient samples for germline testing are accessible from buccal swabs, saliva, or leukocytes obtained from the buffy coat of a peripheral blood sample. Because the germline DNA does not change over time, the timing of testing will not influence the results (as it can for somatic testing). Importantly, a negative family history for cancer does not rule out the presence of a germline mutation, as Pritchard et al. reported a family history of prostate or any cancer in only $22 \%$ and $71 \%$ of positive cases, respectively ${ }^{16}$. While clinically and pathological features may be helpful in increasing the likelihood of identifying a germline mutation $^{17}$, to date no validated model exists to narrow the population of patients at risk of germline DDR gene alterations. As a result, several influential 
international guidelines and consensus statements recommend germline testing of DDR genes be offered to all patients with metastatic prostate cancer, irrespective of other clinical features ${ }^{18-20}$. Despite this recommendation, there is currently no clear route for funding reimbursement for population-based germline testing in prostate cancer.

There are four basic pathways for germline testing: referral to medical geneticists/Hereditary Cancer Clinics (HCC); oncologist mediated genetic testing ("mainstreaming"); direct to consumer commercial testing; and in parallel with somatic testing, particularly circulating tumor DNA (ctDNA) (see discussion below).

\section{Referral for germline genetic testing}

Traditionally, germline genetic testing has been under the auspices of medical geneticists and/or genetic counsellors within HCC. Clinicians refer to HCC based on clinical factors, such as disease characteristics and/or family history. Patients undergo a pre-test evaluation to establish suitability for genetic testing, an appropriate gene panel is selected, and counselling on the potential risks and benefits of genetic testing is performed. Test results are disclosed at a second visit and the patient is counselled on recommended management. This multi-visit model was established when genetic testing was expensive and candidate genes to be tested were relatively few; therefore, it was necessary to restrict testing to individuals meeting strict eligibility criteria, as verified by a trained genetics specialist. This model was also developed in an era without genetic biomarker directed treatment, reducing the urgency for test results. Many patients experience significant delays receiving genetic testing with this model. A recent report from Cancer Care Ontario found that the average wait time for cancer patients from referral to first appointment with a genetic counsellor was 183 calendar days, ranging from 30 to 590 days depending on the $\operatorname{clinic}^{21}$. This model is also inefficient, in that equal time is spent assessing and counselling the minority of patients who are found to have a mutation as the majority who will ultimately not be found to carry a mutation. Furthermore, as the cost of genetic testing has decreased and is now widely availability, the use of a geneticist as gatekeeper may be less important.

\section{Mainstreaming model of germline genetic testing}

First established in the UK (https://www.mcgprogramme.com), the "mainstreaming" model refers to genetic testing ordered by an oncology clinician as part of routine care to address the disadvantages of the traditional model of genetic testing. Importantly, mainstreaming does not take place in isolation, but rather with the support of geneticists and/or HCC, who provide educational resources and accept referrals for individuals with positive findings, or those requiring more in depth counselling ${ }^{22}$. The potential risks to advanced cancer patients are different than healthy individuals. Potential financial risk, such as exclusion from life insurance, loans, and/or mortgages, are not likely to be viewed above and beyond their already established cancer diagnosis. Furthermore, Canada has established the Genetics Non-Discrimination act in 2017, which legally prohibits the requirement of individuals to undergo or disclose the results of 
genetic testing ${ }^{23}$. Importantly, this law was upheld as constitutional by the Supreme Court of Canada in $2020^{24}$. Healthy patients who carry pathogenic or likely pathogenic germline mutations can be faced with complex medical decisions, including more intensive follow-up and screening for cancers, as well as prophylactic procedures, such as mastectomy and oophorectomy. These decisions are not likely to be applicable to a patient with metastatic cancer, as such care would not be appropriate for an individual with a life-limiting illness such as mPC. However, there is still potential emotional and personal consequences to consider. Patients may fear that a pathogenic finding could create a sense of resentment towards them, or they may feel guilt for passing high risk genes to children. While patients are not legally required to disclose the result of genetic testing, they are strongly encouraged to do so, so that family members can seek appropriate genetic testing (cascade testing) and medical care. Finally, mainstreaming has been introduced successfully in a variety of circumstances, including ovarian, breast, and most recently prostate cancer ${ }^{22,25-28}$.

Mainstreaming has demonstrated increased testing rates with decreased wait time compared to the traditional HCC model. For example, in one UK centre only $14 \%$ of eligible patients received germline $B R C A 1 / 2$ testing prior to the implementation of mainstreaming for ovarian cancer, with a mean turnaround time of 148.2 days. This improved to $95 \%$ of eligible patients receiving testing, with a mean turnaround time of 20.6 days with the introduction of mainstreaming $^{25}$. How well mainstreaming would work for $\mathrm{mPC}$ patients in Canada remains to be seen, however an Australian group published their experience of mainstream genetic testing in aPC with $95 \%$ of approached patients consented to testing and high levels of patient and clinician satisfaction ${ }^{28}$. Also, the recent increased utilization of virtual healthcare technologies due to the COVID-19 pandemic represents an opportunity for better geographic diffusion of access to genetic counselling ${ }^{29}$.

As attractive as mainstreaming is, a potential barrier to its widespread adoption is the significant Canadian regional variation in resources and provider comfort in discussing genetic testing. This variation could lead to differences in uptake and application of testing. Mainstreaming also requires a non-insignificant time commitment to both develop the knowledge and expertise required, as well as to obtain consent and initiate genetic testing in clinic. Different models including provider checklists and pre-recording videos can address some of these challenges. This is particularly relevant in community and rural centres where prostate cancer care may represent a small proportion of a broad clinical practice for the clinician. In a health care system that finds many clinicians already overburdened with responsibilities and limited in resources, mainstreaming may not be universally feasible or accepted.

\section{Commercial third-party germline genetic testing}

Patient initiated, clinical grade germline genetic testing through third party private companies is another alternative to the HCC model. Examples include Color Genomics (www.color.com) and Invitae (www.invitae.com). These companies offer rapid testing of saliva samples along with 
post-test genetic counselling. Both companies currently advertise a cost of approximately $\$ 250$ USD, which most Canadians would find affordable. As testing is directly accessible to patients, clinician guidance is strongly encouraged to ensure that an appropriate gene panel is selected and the results are correctly interpreted. While this is an accessible and affordable option for most, many clinicians and patients steeped in the Canadian Health Care system may be uncomfortable at the prospect of facing out-of-pocket costs for what many view should be standard of care. We believe clinicians counselling patients on genetic testing should include this as an option for patients to encourage open and honest dialogue. Furthermore, some clinicians caring for mPC patients may not have ready access to refer a patient to a HCC or necessary resources for mainstreaming, and commercial testing may be the only viable option to access genetic testing. Patients that pursue this should be encouraged to share their results with their health care providers, and if a mutation is identified or there are other concerning features on history, referral to a local HCC (if available) should occur to ensure appropriate counselling, follow up, and referral to local resources takes place. It is important that clinicians and patients are aware of appropriateness of tests ordered as well as reliability of the company selected. There are many direct to consumer (DTC) companies available, but results may not be clinically relevant, complete, or accurate ${ }^{30}$. Some companies include pre and post test genetic counseling; however, and particularly if not included, the clinician should be prepared to discuss this information with their patient.

\section{Somatic testing}

Somatic testing requires access to tumor tissue DNA, which can be acquired from archival biopsy or surgical samples, fresh tumor biopsies, or so called "liquid biopsies" where plasmaderived cell-free DNA (cfDNA) are analyzed from peripheral blood samples. All people have cfDNA in their blood plasma. However, in patients with cancer, a varying proportion of the cfDNA is tumour derived and referred to as circulating tumor DNA (ctDNA). Solid tumor biopsy or surgical samples have long been the standard for somatic testing. As such, many research, clinical, and commercial laboratories are capable of performing tissue based NGS. There are, however, notable disadvantages to this approach in metastatic prostate cancer: The diagnostic or surgical tissue specimen may predate the development of metastatic disease by many years, such that sample availability and quality may not be optimal; many patients that present with de novo metastatic disease are diagnosed clinically with no available tissue sample or by fine needle aspiration (FNA) biopsy, which does not yield sufficient tissue DNA for testing; prostate biopsy can be painful and carries a significant risk of adverse complications ${ }^{31}$; many patients with advanced prostate cancer frequently have bone only metastatic disease, which can be challenging to biopsy, and less likely to yield sufficient material for $\mathrm{NGS}^{32}$; and intratumoral heterogeneity and clonal temporal evolution may not be reflected in a solitary biopsy specimen, though most DNA repair gene alterations appear to be early, truncal events in prostate cancer, with high concordance between diagnostic prostate biopsies and either ctDNA 
samples or metastatic biopsies ${ }^{15,33}$. The somatic testing to determine eligibility in the PROfound clinical trial was performed by Foundation Medicine (Cambridge, MA) on either archival or fresh biopsy formalin-fixed paraffin-embedded (FFPE) samples. The patient disposition seen in PROfound can serve as evidence of the limitations of solid tumor testing: 4425 patients were enrolled for screening, only $2792(63 \%)$ were successfully sequenced, with $378(9 \%)$ not having tissue available, and 1255 (28\%) failing sequencing due to sample inadequacy (predominantly low tumor fraction or DNA yield ${ }^{34}$. However, a recent report from the STAMPEDE trial demonstrated that in samples that were obtained within 8 months, the success rate of tumor based NGS was 92\%, suggesting that the success of tumour based assays is highly dependent on the age of archival tissue, owning to effects of DNA degradation over time ${ }^{35}$.

\section{Circulating tumor DNA somatic testing}

ctDNA is an emerging alternative to tissue-based somatic testing. It is relatively non-invasive, requiring a simple blood draw; intra-patient heterogeneity is potentially less of an issue with ctDNA testing since multiple tumor deposits are presumed to contribute to the ctDNA fraction; and ctDNA can be serially sampled over time due to the minimally-invasive nature, allowing the study of temporal clonal dynamics and resistance mechanisms ${ }^{36,37}$. It is worth noting that similar to the sample inadequacy issues of testing prostate tumor tissue, a significant minority of mCRPC patients have relatively low levels of ctDNA in their blood. The proportion of ctDNA to total cfDNA ("ctDNA fraction") varies significantly depending on the clinical disease state and response to therapy, with patients with progressive mCRPC having higher cfDNA yields and ctDNA fraction than $\mathrm{mHSPC}$ receiving androgen deprivation therapy ${ }^{38}$. Using a threshold ctDNA fraction of $>2 \%$ to be sufficient for somatic testing, approximately $75 \%$ of patients with progressive mCRPC will be successfully tested for somatic mutations using ctDNA ${ }^{39}$. While this ctDNA fraction is sufficient for identifying somatic mutations, identifying copy number alterations require ctDNA fractions of $>10-20 \%$. Clinically available factors associated with disease burden can be used to roughly predict the ctDNA fraction (https://ctdna.org/). The PROfound trial collected ctDNA samples at baseline and were tested by Foundation Medicine (Cambridge, MA) using the FoundationOne Liquid CDx assay, though these results were not used to determine patient eligibility. A total of 619 samples underwent ctDNA testing, with 503 $(81 \%)$ yielding a result, and with a high level of concordance between ctDNA and tissue-based testing. Structural genomic changes, such as homozygous deletions and rearrangements are technically challenging to identify when ctDNA fractions are low, which accounts for some of the discordant results between ctDNA and tumor testing ${ }^{40}$. It should also be noted that ctDNA testing can be confounded by clonal hematopoesis of indeterminate potential, or CHiP. CHiP variants are identified in both whole blood as well as plasma, while ctDNA mutations are only identified in plasma. As such, whole blood leukocyte NGS should accompany plasma NGS, to filter variants that are related to CHiP. Highlighting the importance of this, a recent study in patients with mPC who underwent ctDNA testing found that $10 \%$ had variants in ATM, BRCA2, 
and $C H E K 2$ that were due to $\mathrm{CHiP}^{41}$. Had whole blood leukocyte NGS not been performed, these patients would have falsely been identified as candidates for PARP inhibitor therapy. Therefore, in patients undergoing ctDNA testing, we advise to verify that whole blood leukocyte testing is also being performed to rule out $\mathrm{CHiP}$.

Ultimately, neither tissue- or blood-based somatic testing is able to characterize all patients. As companion biomarker testing for olaparib is being planned and developed, this is an important consideration. While ctDNA testing offers a number of advantages, an optimal diagnostic pathway will be capable of offering both ctDNA and tissue-based testing.

\section{Implementation of DDR testing within the Canadian healthcare system}

The population of $\mathrm{mPC}$ patients requiring germline testing would overwhelm HCC for referrals for pre-test counselling, and we therefore encourage clinicians to initiate discussions with their HCC to identify pathways for referrals, co-education regarding the prevalence of DDR gene alterations, the importance of germline testing, and to discuss the potential to offer mainstreaming. Many centres already offer mainstreaming in some capacity, typically for ovarian and/or breast cancer, and indeed many geneticists prefer this approach. Discussions should also include provincial lab formularies, as the increased demand for germline testing in this population likely result in additional laboratory cost, both at the level of testing as well as result interpretation. However, germline genetic testing has been shown to be cost effective to the health care system as a whole in both ovarian ${ }^{42}$ and breast ${ }^{43}$ cancer and effective risk

reduction measures can be undertaken in asymptomatic carriers ${ }^{44}$. This cost-effectiveness has yet to be explicitly demonstrated in MPC, but it is important to remember that affected men can have carrier female relatives at high risk of breast and ovarian cancer, meaning that $\mathrm{mPC}$ screening also offers a strategy to further reduce the mortality of breast and ovarian cancers.

Access to somatic testing varies regionally. To our knowledge, no Canadian jurisdiction currently provides provincial reimbursement for somatic NGS in mPC. However, a number of institutions have research protocols which provide somatic NGS for patients with advanced cancer, such as OCTANE (Ontario-wide Cancer TArgeted Nucleic acid Evaluation, ClinicalTrials.gov Identifier: NCT02906943) ${ }^{45}$ or the Personalized Oncogenomics (POG) Program of British Columbia (ClinicalTrials.gov Identifier: NCT02155621). Other institutions may be able to access somatic NGS for select patients through interventional clinical trials, as a number of actively recruiting trials offer this as a screening requirement. Examples include the Canadian Clinical Trials Group PC-BETS trial (ClinicalTrials.gov Identifier: NCT03385655) or TRITON3 (ClinicalTrials.gov Identifier: NCT02975934). Despite this, for the majority of Canadian prostate cancer patients the only way to access somatic NGS is through third party commercial testing. Companies such as Foundation Medicine (www.foundationmedicine.com), which offers both tissue- and ctDNA-based somatic testing, and Caris Life Sciences (www.carislifesciences.com), which offers tissue-based testing, are available to patients who are able and willing to pay out-of-pocket. The cost of somatic testing through third party commercial 
testing, which typically ranges in the thousands of dollars, will be prohibitive for many patients. As such, until there is provincial reimbursement for companion diagnostic somatic NGS for advanced prostate cancer patients, access to testing will be limited. Frank and honest discussions with patients regarding this will be required, along with a knowledge of how to access testing for patients who wish to do so.

Germline and somatic testing should be viewed as complimentary and ideally not performed in isolation. Somatic-only NGS does not differentiate between somatic and germline mutations, although computational methods can be used when analyzing tissue based somatic NGS results that are highly predictive for germline mutations ${ }^{46}$. These methods may not be done by all labs, and therefore pathogenic/likely pathogenic (Tier 1 or 2) (table 2) somatic testing results will usually also require germline testing to direct the need for cascade testing of at risk family members. While somatic testing is sufficient for the purposes of directing PARP inhibitor therapy, germline testing is not, with less than half of actionable BRCA1, BRCA2, and ATM alterations detected on tumor testing in MCRPC found to be germline in origin ${ }^{12}$.

\section{Provincial reimbursement}

A decision on provincial reimbursement for olaparib and a companion diagnostic (in this case somatic NGS) is pending at the time of publication, though a positive decision seems likely given the magnitude of benefit seen, the novel mechanism of action, as well as precedence of approval in other diseases, namely ovarian cancer ${ }^{47}$. The exact type and ideal pathway of testing is yet to be determined and will likely vary between regions. We encourage clinicians to engage with their molecular pathology labs and clinical genetics departments to begin discussions regarding provincial reimbursement and to define optimal local pathways for testing.

\section{Management of the metastatic prostate cancer patient with DDR gene alterations}

\section{Patient selection for olaparib}

While olaparib is Health Canada approved for use in mCRPC patients with $B R C A 1, B R C A 2$, and $A T M$ alterations based on the landmark PROfound clinical trial ${ }^{10,35}$, emerging evidence suggests that these three genes may confer varying degrees of sensitivity to PARP inhibitors.

\section{BRCA2}

$B R C A 2$ is the most frequently altered DNA repair gene in $\mathrm{mPC}$ and represents the largest population of patients in the PROfound trial, making up 52\% of Cohort A, and $33 \%$ of the overall treatment population ${ }^{35}$. In contrast, $B R C A 1$ mutations are infrequent in advanced prostate cancer, occurring in approximately $1 \%$ of mCRPC patients ${ }^{12}$, and only $5 \%$ of patients in Cohort A of PROfound. This is in contrast to other cancers where PARP inhibitors have received regulatory approval, such as ovarian ${ }^{48}{\text {, } \text { breast }^{49} \text {, and pancreatic }}^{50}$ cancer, where both $B R C A 1$ and $B R C A 2$ represent significant patient populations. In the subgroup analysis of the PROfound trial, as well as an exploratory gene-by-gene analysis, BRCA1 and BRCA2 are grouped together ${ }^{35,51}$. 
As such, no conclusions on the relative efficacy of olaparib in these gene mutations can be drawn from this trial. However, given the preponderance of $B R C A 2$, we can assume that these patients are responsible for the majority of the effect in this analysis. Given an objective response rate of $44 \%$, PSA response rate of $62 \%$, and robust improvements in rPFS and OS, it is clear that olaparib is highly active in BRCA2 mutated mCRPC.

\section{BRCA1}

It is challenging to draw conclusions regarding the activity of olaparib in BRCA1 mutated mCRPC from PROfound; however, the phase II TRITON2 study, which investigated the efficacy of rucaparib in mCRPC patients with $B R C A 1$ or $B R C A 2$ mutations, identified a lower PSA response rate in $B R C A 1$ compared to $B R C A 2$, at $15.4 \%$ and $59.8 \%$, respectively, as well as lower ORR and shorter PFS $^{52}$. Similarly, a retrospective multi-centre study of 123 BRCA1 or BRCA2 altered mCRPC patients treated with PARP inhibitors found a significantly lower PSA response rate, and shorter PFS and OS for $B R C A 1$ compared to $B R C A 2^{53}$. Given that olaparib is known to be highly active in other malignancies with $B R C A 1$ mutations, it is tempting to dismiss these results; however, there may be biological rationale to explain this differential sensitivity to PARP inhibition. A pan-cancer analysis of 234,154 tumors sequenced by Foundation Medicine found that the vast majority of $B R C A 1$ alterations in prostate cancer were mono-allelic (meaning that there is a still a functional allele), whereas the vast majority of $B R C A 2$ alterations were biallelic $^{54}$. While there is still clearly activity of PARP inhibitors in BRCA1 altered mCRPC, the benefit may be less relative to $B R C A 2$ and may require a more nuanced patient identification based on bi-allelic loss. Further evidence is required to fully characterize the activity and optimize patient selection of PARP inhibitors in these patients.

\section{ATM}

An exploratory gene-by-gene analysis of the PROfound trial demonstrated that for patients with ATM mutations both median rPFS and OS were similar to the control arm, with HR of 1.04 and 0.93 , respectively ${ }^{51}$. The objective response rate was $10 \%$ in both the olaparib and control arms, with confirmed PSA response in fact lower in ATM patients who received olaparib. The TOPARP-B study reported a composite response rate of $36.8 \%$ of patients with ATM alterations, but it is important to point out that the majority of these responses were achieved due to circulating tumor cell (CTC) conversion, a response criterion not typically used in clinical practice $^{55}$. The PSA and objective response rates from this study, 5.3\% and $8.3 \%$ respectively, are similar to what was reported in PROfound. Furthermore, the phase II TRITON2 study found similarly modest activity of rucaparib in $A T M$ altered $\mathrm{mCRPC}^{56}$. As the PROfound trial was not designed to investigate the benefit of olaparib based on individual genes or prior taxane use, caution must be exercised in interpreting these results. However, it is clear that ATM alterations appear to confer less sensitivity to PARP inhibitors than BRCA2.

\section{Treatment sequencing}


Where olaparib will fit into the treatment sequence for a patient with mCRPC is not well defined. The treatment population for PROfound mandated at least one ARAT and allowed all approved mCRPC therapies; therefore, patients could have had as few as 1, or as many as 6 life prolonging mCRPC therapies prior to study entry. The Health Canada approval mandates one prior ARAT, but is agnostic on what disease state (mCRPC, mCSPC, nmCRPC) it has been used, as well as other prior lines of therapy. Additionally, there is insufficient evidence to conclude whether $B R C A 1 / 2$ or other DNA repair gene mutations are predictive of response (or lack thereof) to other standard of care therapies such as ARAT and taxanes. Studies examining this are challenging to interpret due to the heterogeneity in patient populations studied, therapies used, and definitions of benefit. These studies have reported worse $e^{57,58}, \operatorname{similar}^{14,59}$ or even improved outcomes $^{60}$ in DDR gene altered prostate cancers relative to controls. Based on this one cannot draw any meaningful conclusions. Lacking high quality evidence, the default position is that these patients should still be considered for all potential therapies, given that the registration trials for these life-prolonging treatments did not utilize biomarker selection. Ultimately, the decision for where to use olaparib in the treatment sequence rests with the physician and patient. Below are considerations that may guide these decisions.

\section{Second-line ARAT}

The preference for olaparib over second-line ARAT is clearly present, as this was the comparator arm of the PROfound study, over which olaparib demonstrated superiority. It is our opinion that this preference remains irrespective of the disease context in which the initial ARAT was prescribed. For example, the patient who received their initial ARAT in nmCRPC or mCSPC should be viewed the same as a patient who received their initial ARAT in MCRPC. There is no biological rationale that the disease context of initial therapy affects response to further therapy, and sequential ARAT therapy in $\mathrm{mCRPC}$ has demonstrated very modest clinical activity ${ }^{61}$.

\section{Docetaxel}

The Health Canada approval for docetaxel in mCRPC was made based on the TAX327 study, which predated the development of abiraterone and enzalutamide ${ }^{2}$ and therefore not representative of the patients treated in the modern era. However, retrospective series have shown docetaxel remains active after progression on $\mathrm{ARAT}^{62,63}$. In the specific population of $B R C A 1, B R C A 2$ or ATM altered mCRPC, available evidence generally indicate that docetaxel has similar efficacy compared to wildtype mCRPC patients ${ }^{58,59}$, though one series reported worse cancer specific survival after first taxane in germline BRCA2 patients compared to controls, despite similar response rates ${ }^{58}$. Alternatively, at least for $B R C A 2$ altered mCRPC patients, there is robust survival data for olaparib in a modern patient population, with high PSA and RECIST responses. However, in the absence of direct a head to head comparison, both agents are reasonable options. When considering BRCAl and ATM, the choice is somewhat more complicated, given the smaller patient numbers represented in PROfound, and less impressive outcomes in sub-group analyses from PROfound and TRITON2, as discussed above. Thus, in 
$B R C A 1$ and $A T M$ altered patients a clear preference of olaparib or docetaxel is not present, and will ultimately depend on clinician and patient preferences, though some authors have advocated a preference for taxanes over olaparib in non-BRCA2 altered cancers ${ }^{64}$. Of note, the ongoing TRITON3 study (ClinicalTrials.gov Identifier: NCT02975934) investigating rucaparib versus investigator's choice, includes the option for docetaxel in the control arm, providing a direct comparison of a PARP inhibitor vs docetaxel in DDR gene altered mCRPC.

\section{Cabazitaxel}

For a patient who has received prior ARAT and docetaxel, and is a candidate for further cytotoxic chemotherapy, the choice between cabazitaxel and olaparib is present. Similar to docetaxel, the registration trial that led to the approval of cabazitaxel occurred in an era where the vast majority of patients had not received an ARAT ${ }^{3}$. However the CARD trial provides recent prospective data that confirms the survival advantage of cabazitaxel post-docetaxel in a ARAT pre-treated patients ${ }^{65}$. Therefore both cabazitaxel and olaparib have high quality evidence of survival data in a representative patient population. The CARD trial did not have any biomarker selection or stratification, therefore one cannot draw conclusions on the impact of $B R C A 2$ or other DDR gene mutations on the efficacy of cabazitaxel. In the absence of comparative data, both are acceptable options. Choice of therapy should be driven by patients factors, including which qualifying mutation is present.

\section{Radium-223}

Not all Canadian provinces reimburse radium-223 and is therefore not universally available. Interestingly, emerging evidence suggests that mCRPC patients with DDR genes alterations may derive a greater benefit from radium-223 than DDR gene wildtype patients. A multicentre retrospective study demonstrated a significantly improved overall survival in patients with DDR altered compared to wildtype mCRPC patients ${ }^{66}$, as well as an interim analysis from the prospective PRORADIUM study similarly demonstrated a trend towards improved OS ${ }^{67}$. Once again, in the absence of a direct head-to-head comparison it is difficult to make recommendations regarding a preference of olaparib versus radium-223, as both are supported by overall survival benefit in high quality randomized controlled trials. The choice will ultimately depend on treatment availability along with clinician and patient preferences.

\section{Platinum-based chemotherapy}

While no platinum-based chemotherapy has received Health Canada approval for prostate cancer, many clinicians consider it a reasonable option in select clinical circumstances. Several recent retrospective series have shown that platinum-based chemotherapy is active in DDR gene altered mCRPC ${ }^{68,69}$, with comparable PSA and soft tissue responses in BRCA2 patients as was reported in PROfound ${ }^{70}$, and responses even after progression on PARP inhibitors. While platinum-based chemotherapy appears to be active in this patient population, olaparib is preferable given that it has demonstrated an OS benefit in a phase III randomized control trial, a 
level of evidence that doesn't exist for platinum. However, in patients who cannot access or who have progressed on olaparib, platinum-based chemotherapy is an option.

\section{Future directions}

While germline testing for prostate cancer has been endorsed by international guidelines and has been adopted in many centres, to date very little research has been published on the impact on patients, their families, and healthcare systems. To our knowledge, there have been no publications on cost-effectiveness of germline testing in prostate cancer, representing an area of unmet need. The optimal testing algorithm and time to test patients for somatic and germline DNA repair gene alterations is unclear. At present the only indication for somatic testing is to determine eligibility for olaparib after progression on at least one ARAT. However, germline testing is ideally done as soon as possible to inform at risk family members so that they can access appropriate screening and management. Also, it seems likely that PARP inhibitor therapy may move into earlier disease states, as is currently being investigated in a number of clinical trials. We anticipate that testing algorithms will evolve and likely suggest earlier initiation of testing. A number of questions still remain on which patients are most likely to benefit from PARP inhibitors. As other trials are published, along with real world datasets, we expect to see a refinement in which specific DNA repair gene alterations are predictive of response. We may see also a move away from identifying specific gene alterations, and rather a phenotypic analysis, such as Myriad Genetics myChoice, which has been used in some ovarian cancer trials ${ }^{71}$. This is appealing, as rather than predicting whether an HR defect is present based on alterations of individual genes, these scores provide an assessment of genomic alterations that associates with defective HR.

While olaparib is the first PARP inhibitor to receive Health Canada approval, a number of other agents, including rucaparib, niraparib, and talazoparib, are undergoing clinical trials and may also receive regulatory approval. It is likely that PARP inhibitors will move into earlier disease states, as has occurred with many other agents that received their initial approval in pretreated mCRPC patients. Phase III trials are already underway investigating PARP inhibitors in other settings including mCSPC, first line $\mathrm{mCRPC}$, and pre-treated mCRPC, with most combining a PARP inhibitor with other systemic therapies [Table 3]. This includes standard of care $\mathrm{mPC}$ therapies, such as abiraterone and enzalutamide, based on supporting pre-clinical evidence as well as a randomized phase II study that demonstrated a benefit of olaparib in combination with abiraterone regardless of homologous recombination repair mutation status ${ }^{72}$. PARP inhibitors in combination with other DNA repair pathway inhibitors, such as ATR, is also under active investigation. This combination has encouraging pre-clinical rationale for both $B R C A$ and $A T M$ altered cancers ${ }^{73,74}$. Recently the combination of olaparib with cediranib, a small molecule VEGFR TKI, demonstrated improved rPFS over olaparib monotherapy in mCRPC 
patients, which appeared to be greatest in homologous recombination deficient cancers, particularly those with non-BRCA2 DNA repair gene alterations ${ }^{75}$.

\section{Conclusions}

The Health Canada approval of olaparib has introduced a new biomarker driven era in the management of advanced prostate cancer. This brings the prospect of both challenges and opportunities. Clinicians will need to become familiar with the nuances of accessing and interpreting both germline and somatic testing. Clinicians, geneticists, and molecular pathologists must also play a role in advocating for patients to establish streamlined routes for such testing along with provincial reimbursement. While this biomarker defined patient population has only recently been identified, we are seeing a rapid emergence of literature defining the clinical characteristics of these patients. We expect to see a refinement in patient identification, use of standard of care therapies (including platinum based chemotherapy), as well as novel therapies in combination with PARP inhibitors. 


\section{References}

1. Huggins C and Hodges C. Studies on Prostatic Cancer. I. The Effect of Castration, of Estrogen and of Androgen Injection on Serum Phosphatases in Metastatic Carcinoma of the Prostate. Cancer Res April 11941 (1) (4) 293-297.

2. Tannock IF, de Wit R, Berry WR, et al. Docetaxel plus prednisone or mitoxantrone plus prednisone for advanced prostate cancer. N Engl J Med 2004 Oct 7;351(15):1502-12.

3. de Bono JS, Oudard S, Ozguroglu M, et al. Prednisone plus cabazitaxel or mitoxantrone for metastatic castration-resistant prostate cancer progressing after docetaxel treatment: a randomised open-label trial. Lancet 2010 Oct 2;376(9747):1147-54.

4. de Bono JS, Logothetis CJ, Molina A, et al. Abiraterone and increased survival in metastatic prostate cancer. N Engl J Med 2011 May 26;364(21):1995-2005.

5. Scher HI, Fizazi K, Saad F, et al. Increased survival with enzalutamide in prostate cancer after chemotherapy. N Engl J Med 2012 Sep 27;367(13):1187-97.

6. Chi KN, Agarwal N, Bjartell A, et al. Apalutamide for Metastatic, Castration-Sensitive Prostate Cancer. N Engl J Med 2019 Jul 4;381(1):13-24.

7. Fizazi K, Shore N, Tammela TL, et al. Nonmetastatic, Castration-Resistant Prostate Cancer and Survival with Darolutamide. N Engl J Med 2020 Sep 10;383(11):1040-1049.

8. Parker C, Nilsson S, Heinrich D, et al. Alpha emitter radium-223 and survival in metastatic prostate cancer. N Engl J Med 2013 Jul 18;369(3):213-23.

9. Kantoff PW, Higano CS, Shore ND, et al. Sipuleucel-T immunotherapy for castrationresistant prostate cancer. N Engl J Med 2010 Jul 29;363(5):411-22.

10. Hussain M, Mateo J, Fizazi K, et al. Survival with Olaparib in Metastatic CastrationResistant Prostate Cancer. N Engl J Med 2020 Dec 10;383(24):2345-57.

11. Sartor O, de Bono J, Chi KN, et al. Lutetium-177-PSMA-617 for Metastatic CastrationResistant Prostate Cancer. N Engl J Med 2021 Jun 23. Epub ahead of print.

12. Robinson D, Van Allen EM, Wu YM, et al. Integrative clinical genomics of advanced prostate cancer. Cell 2015 May 21;161(5):1215-1228.

13. Abida W, Armenia J, Gopalan A, et al. Prospective Genomic Profiling of Prostate Cancer Across Disease States Reveals Germline and Somatic Alterations That May Affect Clinical Decision Making. JCO Precis Oncol 2017 Jul;2017:PO.17.00029.

14. Abida W, Cyrta J, Heller G, et al. Genomic correlates of clinical outcome in advanced prostate cancer. Proc Natl Acad Sci U S A 2019 Jun 4;116(23):11428-11436.

15. Warner E, Herberts C, Fu S, et al. BRCA2, ATM, and CDK12 Defects Differentially Shape Prostate Tumor Driver Genomics and Clinical Aggression. Clin Cancer Res 2021 Jan 7. Epub ahead of print.

16. Pritchard CC, Mateo J, Walsh MF, et al. Inherited DNA-Repair Gene Mutations in Men with Metastatic Prostate Cancer. N Engl J Med 2016 Aug 4;375(5):443-53.

17. Yip S, Sunderland K, Beigi A, et al. Predictors of germline DNA damage repair gene mutations (gDDRm) in patients (pts) with metastatic castration-resistant prostate cancer (CRPC). J Clin Oncol 37, no. 7_suppl (March 01, 2019) 159-159.

18. Mohler JL, Srinivas S, Antonarakis ES, et al: Prostate cancer, version 1.2020, NCCN clinical practice guidelines in oncology. J Natl Compr Canc Netw 17: 479-505, 2020. 
19. Giri VN, Knudsen KE, Kelly WK, et al. Role of Genetic Testing for Inherited Prostate Cancer Risk: Philadelphia Prostate Cancer Consensus Conference 2017. J Clin Oncol 2018 Feb 1;36(4):414-424.

20. Gillessen S, Attard G, Beer TM, et al. Management of Patients with Advanced Prostate Cancer: Report of the Advanced Prostate Cancer Consensus Conference 2019. Eur Urol 2020 Apr;77(4):508-547.

21. Cancer Care Ontario. Recommendation Report for Ontario's Clinical Genetic Services. May 2018. Available at: https://www.cancercareontario.ca/sites/ccocancercare/files/assets/ClinicalGeneticServices RecommendationReport.pdf. Accessed 28 May 2021.

22. George A, Riddell D, Seal S, et al. Implementing rapid, robust, cost-effective, patientcentred, routine genetic testing in ovarian cancer patients. Sci Rep 2016 Jul 13;6:29506.

23. canada.ca: Genetic Non-Discrimination Act (S.C. 2017, c. 3): https://lawslois.justice.gc.ca/eng/acts/G-2.5/index.html. Access 28 May 2021.

24. cbc.ca: Supreme Court of Canada upholds genetic non-discrimination law [posted $10 \mathrm{Jul}$ 2020]: https://www.cbc.ca/news/politics/stefanovich-supreme-court-of-canada-geneticinformation-1.5643245. Accessed 28 May 2021.

25. Rumford M, Lythgoe M, McNeish I, et al. Oncologist-led BRCA 'mainstreaming' in the ovarian cancer clinic: A study of 255 patients and its impact on their management. Sci Rep 2020 Feb 25;10(1):3390.

26. Wong E, Chrystal K, Wilson M, et al. Implementation of mainstream BRCA testing in epithelial ovarian cancer in a tertiary centre. Ann Oncol Volume 30, SUPPLEMENT 9, ix81, November 01, 2019.

27. Kemp Z, Turnbull A, Yost S, et al. Evaluation of Cancer-Based Criteria for Use in Mainstream BRCA1 and BRCA2 Genetic Testing in Patients With Breast Cancer. JAMA Netw Open 2019 May 3;2(5):e194428.

28. Scheinberg T, Goodwin A, Ip E, et al. Evaluation of a Mainstream Model of Genetic Testing for Men With Prostate Cancer. JCO Oncol Pract 2021 Feb;17(2):e204-e216.

29. Glauser W. Virtual care is here to stay, but major challenges remain. CMAJ 2020 Jul 27;192(30):E868-E869.

30. Nelson TN, Armstrong L, Richer J, et al. CCMG Statement on Direct-to-Consumer Genetic Testing. https://www.ccmgccgm.org/documents/Policies_etc/Pos_Statements/PosStmt_EPP_DTC_FINAL_20Jan201 1.pdf. Accessed 16 Sep 2021.

31. Liss MA, Ehdaie B, Loeb S, et al. An Update of the American Urological Association White Paper on the Prevention and Treatment of the More Common Complications Related to Prostate Biopsy. J Urol 2017 Aug;198(2):329-334.

32. Spritzer CE, Afonso PD, Vinson EN, et al. Bone marrow biopsy: RNA isolation with expression profiling in men with metastatic castration-resistant prostate cancer--factors affecting diagnostic success. Radiology 2013 Dec;269(3):816-23.

33. Mateo J, Seed G, Bertan C. Genomics of lethal prostate cancer at diagnosis and castration resistance. J Clin Invest 2020 Apr 1;130(4):1743-1751.

34. de Bono J, Mateo J, Fizazi K, et al. Olaparib for Metastatic Castration-Resistant Prostate Cancer. N Engl J Med 2020 May 28;382(22):2091-2102. 
35. Gilson C, Ingleby F, Gilbert DC, et al. Genomic Profiles of De Novo High- and LowVolume Metastatic Prostate Cancer: Results From a 2-Stage Feasibility and Prevalence Study in the STAMPEDE Trial. JCO Precision Oncology no. 4 (2020) 882-897.

36. Quigley D, Alumkal JJ, Wyatt AW, et al. Analysis of Circulating Cell-Free DNA Identifies Multiclonal Heterogeneity of BRCA2 Reversion Mutations Associated with Resistance to PARP Inhibitors. Cancer Discov 2017 Sep;7(9):999-1005.

37. Cheng HH, Salipante SJ, Nelson PS, et al. Polyclonal BRCA2 Reversion Mutations Detected in Circulating Tumor DNA After Platinum Chemotherapy in a Patient With Metastatic Prostate Cancer. JCO Precis Oncol 2018 Feb 14;2:PO.17.00169.

38. Kohli M, Tan W, Zheng T, et al. Clinical and genomic insights into circulating tumor DNA-based alterations across the spectrum of metastatic hormone-sensitive and castrateresistant prostate cancer. EBioMedicine 2020 Apr;54:102728.

39. Wyatt AW, Annala M, Aggarwal R, et al. Concordance of Circulating Tumor DNA and Matched Metastatic Tissue Biopsy in Prostate Cancer. J Natl Cancer Inst 2017 Dec $1 ; 109(12): \operatorname{djx} 118$.

40. Chi KN, Barnicle A, Sibilla C, et al. Concordance of BRCA1, BRCA2 (BRCA), and ATM mutations identified in matched tumor tissue and circulating tumor DNA (ctDNA) in men with metastatic castration-resistant prostate cancer (mCRPC) screened in the PROfound study. J Clin Oncol 39, 2021 (suppl 6; abstr 26).

41. Jensen K, Konnick EQ, Schweizer MT, et al. Association of Clonal Hematopoiesis in DNA Repair Genes With Prostate Cancer Plasma Cell-free DNA Testing Interference. JAMA Oncol 2021 Jan 1;7(1):107-110.

42. Eccleston A, Bentley A, Dyer M, et al. A Cost-Effectiveness Evaluation of Germline BRCA1 and BRCA2 Testing in UK Women with Ovarian Cancer. Value Health 2017 Apr;20(4):567-576.

43. Tuffaha HW, Mitchell A, Ward RL, et al. Cost-effectiveness analysis of germ-line BRCA testing in women with breast cancer and cascade testing in family members of mutation carriers. Genet Med 2018 Sep;20(9):985-994.

44. Paluch-Shimon S, Cardoso F, Sessa C, et al. Prevention and screening in BRCA mutation carriers and other breast/ovarian hereditary cancer syndromes: ESMO Clinical Practice Guidelines for cancer prevention and screening. Ann Oncol 2016 Sep;27(suppl 5):v103v110.

45. Malone ER, Saleh RR, Yu C, et al. OCTANE (Ontario-wide Cancer Targeted Nucleic Acid Evaluation): a platform for intraprovincial, national, and international clinical data-sharing. Curr Oncol 2019 Oct;26(5):e618-e623.

46. Sun JX, He Y, Sanford E, et al. A computational approach to distinguish somatic vs. germline origin of genomic alterations from deep sequencing of cancer specimens without a matched normal. PLOS Computational Biology 2018; 14: e1005965.

47. albertahealthservices.ca. Laboratory Bulletin re: Next generation sequencing BRCA1/2 tumor molecular panel [uploaded 2020 May 4]. https://www.albertahealthservices.ca/assets/wf/lab/wf-lab-bulletin-next-generationsequencing-brca1-2-tumor-molecular-panel.pdf. Accessed 19 May 2021.

48. Moore K, Colombo N, Scambia G, et al. Maintenance Olaparib in Patients with Newly Diagnosed Advanced Ovarian Cancer. N Engl J Med 2018 Dec 27;379(26):2495-2505. 
49. Robson M, Im SA, Senkus E, et al. Olaparib for Metastatic Breast Cancer in Patients with a Germline BRCA Mutation. N Engl J Med 2017 Aug 10;377(6):523-533.

50. Golan T, Hammel P, Reni M, et al. Maintenance Olaparib for Germline BRCA-Mutated Metastatic Pancreatic Cancer. N Engl J Med 2019 Jul 25;381(4):317-327.

51. de Bono JS, Matsubara N, Penel N, et al. Exploratory gene-by-gene analysis of olaparib in patients (pts) with metastatic castration-resistant prostate cancer (mCRPC): PROfound. J Clin Onco 39, no. 6_suppl (February 20, 2021) 126-126.

52. Abida W, Patnaik A, Campbell D, et al. Rucaparib in Men With Metastatic CastrationResistant Prostate Cancer Harboring a BRCA1 or BRCA2 Gene Alteration. J Clin Oncol 2020 Aug 14:JCO2001035.

53. Taza F, Holler AE, Adra N, et al. Differential activity of PARP inhibitors in BRCA1versus BRCA2-altered metastatic castration-resistant prostate cancer (mCRPC). J Clin Oncol 39, no. 6_suppl (February 20, 2021) 100-100.

54. Sokol E, Pavlick D, Khiabanian H, et al. Pan-Cancer Analysis of BRCA1 and BRCA2 Genomic Alterations and Their Association With Genomic Instability as Measured by Genome-Wide Loss of Heterozygosity. JCO Precision Oncology no. 4 (2020) 442-465.

55. Mateo J, Porta N, Bianchini D, et al. Olaparib in patients with metastatic castrationresistant prostate cancer with DNA repair gene aberrations (TOPARP-B): a multicentre, open-label, randomised, phase 2 trial. Lancet Oncol. 2020 Jan;21(1):162-174.

56. Abida W, Campbell D, Patnaik A, et al. Non-BRCA DNA Damage Repair Gene Alterations and Response to the PARP Inhibitor Rucaparib in Metastatic CastrationResistant Prostate Cancer: Analysis From the Phase II TRITON2 Study. Clin Cancer Res 2020 Jun 1;26(11):2487-2496.

57. Annala M, Vandekerkhove G, Khalaf D, et al. Circulating Tumor DNA Genomics Correlate with Resistance to Abiraterone and Enzalutamide in Prostate Cancer. Cancer Discov 2018 Apr;8(4):444-457.

58. Castro E, Romero-Laorden N, Del Pozo A, et al. PROREPAIR-B: A Prospective Cohort Study of the Impact of Germline DNA Repair Mutations on the Outcomes of Patients With Metastatic Castration-Resistant Prostate Cancer. J Clin Oncol 2019 Feb 20;37(6):490-503.

59. Mateo J, Cheng HH, Beltran H, et al. Clinical Outcome of Prostate Cancer Patients with Germline DNA Repair Mutations: Retrospective Analysis from an International Study. Eur Urol 2018 May;73(5):687-693.

60. Antonarakis ES, Lu C, Luber B, et al. Germline DNA-repair Gene Mutations and Outcomes in Men with Metastatic Castration-resistant Prostate Cancer Receiving First-line Abiraterone and Enzalutamide. Eur Urol 2018 Aug;74(2):218-225.

61. Khalaf DJ, Annala M, Taavitsainen S, et al. Optimal sequencing of enzalutamide and abiraterone acetate plus prednisone in metastatic castration-resistant prostate cancer: a multicentre, randomised, open-label, phase 2, crossover trial. Lancet Oncol 2019 Dec;20(12):1730-1739.

62. Suzman DL, Luber B, Schweizer MT, et al. Clinical activity of enzalutamide versus docetaxel in men with castration-resistant prostate cancer progressing after abiraterone. Prostate 2014 Sep;74(13):1278-85.

63. de Bono JS, Smith MR, Saad F, et al. Subsequent Chemotherapy and Treatment Patterns After Abiraterone Acetate in Patients with Metastatic Castration-resistant Prostate Cancer: Post Hoc Analysis of COU-AA-302. Eur Urol 2017 Apr;71(4):656-664. 
64. Robinson AG, Izard JP, Vera-Badillo FE. Treatment and Patient Selection for Patients with Metastatic Castration-resistant Prostate After Progression on Docetaxel and Abiraterone/Enzalutamide: When to Play Your CARD and When to Do Your PARP. Eur Urol 2021 Mar 25:S0302-2838(21)00172-X.

65. de Wit R, de Bono J, Sternberg CN, et al. Cabazitaxel versus Abiraterone or Enzalutamide in Metastatic Prostate Cancer. N Engl J Med 2019 Dec 26;381(26):2506-2518.

66. van der Doelen MJ, Isaacsson Velho P, Slootbeek PHJ, et al. Impact of DNA damage repair defects on response to radium-223 and overall survival in metastatic castrationresistant prostate cancer. Eur J Cancer 2020 Sep;136:16-24.

67. Castro E, Lozano Mejorada R, Saez M, et al. Impact of germline mutations in homologous recombination (HR) genes on the response to Radium-223 for metastatic castration resistant prostate cancer (mCRPC). Ann Oncol Volume 30, Supplement 5, October 2019, Pages v343-v344.

68. Kwon DH, Chou J, Yip SM, et al. Differential Treatment Outcomes in BRCA1/2-, CDK12-, and ATM-Mutated Metastatic Castration-Resistant Prostate Cancer. Cancer. 2021 Jun 15;127(12):1965-1973.

69. Mota JM, Barnett E, Nauseef JT, et al. Platinum-Based Chemotherapy in Metastatic Prostate Cancer With DNA Repair Gene Alterations. JCO Precis Oncol 2020;4:355-366.

70. Schmid S, Omlin A, Higano C, et al. Activity of Platinum-Based Chemotherapy in Patients With Advanced Prostate Cancer With and Without DNA Repair Gene Aberrations. JAMA Netw Open 2020 Oct 1;3(10):e2021692.

71. González-Martín A, Pothuri B, Vergote I, et al. Niraparib in Patients with Newly Diagnosed Advanced Ovarian Cancer. N Engl J Med 2019 Dec 19;381(25):2391-2402.

72. Clarke N, Wiechno P, Alekseev B, et al. Olaparib combined with abiraterone in patients with metastatic castration-resistant prostate cancer: a randomised, double-blind, placebocontrolled, phase 2 trial. Lancet Oncol 2018 Jul;19(7):975-986.

73. Kim H, George E, Ragland R, et al. Targeting the ATR/CHK1 Axis with PARP Inhibition Results in Tumor Regression in BRCA-Mutant Ovarian Cancer Models. Clin Cancer Res 2017 Jun 15;23(12):3097-3108.

74. Jette NR, Kumar M, Radhamani S, et al. ATM-Deficient Cancers Provide New Opportunities for Precision Oncology. Cancers (Basel) 2020 Mar 14;12(3):687.

75. McKay RR, Radke MR, Shyr Y, et al. Biomarker analysis from a randomized phase II study of olaparib with or without cediranib in men with metastatic castration-resistant prostate cancer (mCRPC). J Clin Oncol 39, 2021 (suppl 6; abstr 7). 


\section{Figures and Tables}

Fig. 1. A schematic representation of mainstreaming germline genetic testing compared to the traditional pathway of referral to a Hereditary Cancer Clinic (HCC).

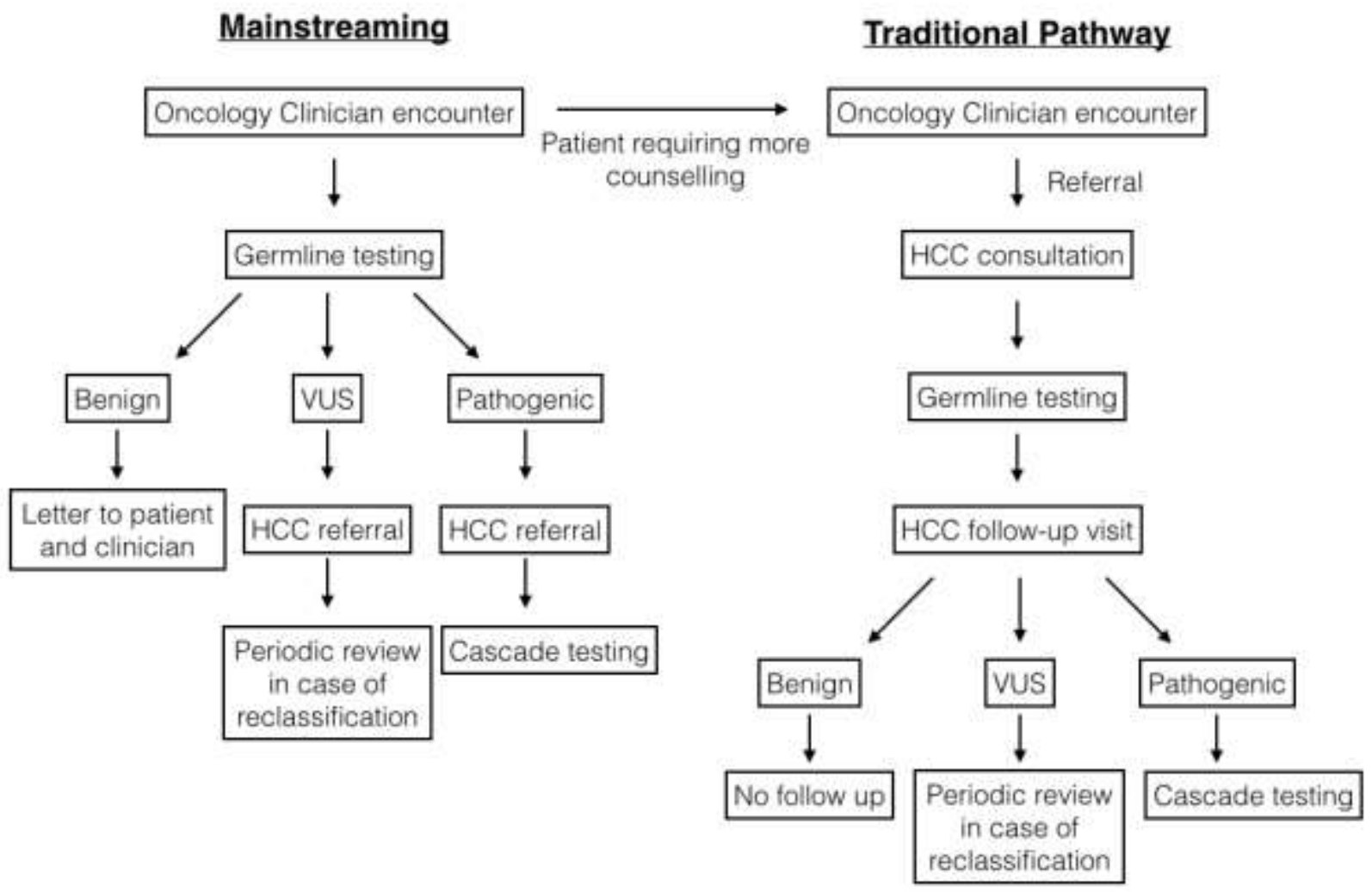




\section{Table 1. Glossary of select terminology}

ATM gene: located on chromosome 11, the ataxia-telangiectasia mutated gene encodes a protein essential in the detection and signaling to repair DNA double-strand breaks. While it is considered a DNA damage repair gene, it does not appear to have any direct role in homologous recombination repair.

BRCA genes: the BRCA1 (chromosome 17) and BRCA2 (chromosome 13) genes encode proteins responsible for homologous recombination repair of double-strand DNA breaks. Functioning as tumour suppressor genes, deleterious BRCA mutations can result in dysregulated cell growth and cell replication. Similar to other tumour suppressor genes (e.g., TP53, RB1), loss of a single copy (monoallelic loss) of BRCA1/BRCA2 is not considered sufficient to lead to an adverse phenotype, owing to restoration of adequate protein function by the remaining functional copy of the gene.

Cell-free DNA: high-fragmented DNA that is released from cells through apoptosis, necrosis and/or active secretion. Cell-free DNA has been most closely studied in the blood, but can exist in various other bodily fluids, including urine, pleural fluid and peritoneal fluid.

Circulating tumour DNA fraction (ctDNA \%): in patients with advanced cancer, the blood contains an admixture of cell-free DNA derived from malignant and non-malignant sources. Next-generation sequencing of patient plasma can provide an estimate of the proportion of total cell-free DNA that is tumour derived, known as the ctDNA fraction. CtDNA\% has been found to be prognostic and predictive in multiple tumour types, including advanced prostate cancer.

Clonal haematopoiesis of indeterminate potential (CHIP) mutations: CHIP mutations represent clonal expansion of white blood cells derived from hematopoietic stem/progenitor cells that possess one or more somatic mutations. Incidence rises with age, and occurs in the absence of any detectable haematologic malignancy. Failure to account for CHIP mutations (by concurrent sequencing of white blood cells) can result in false-positive detection of somatic alterations. This is particularly relevant in advanced prostate cancer, where CHIP mutations have been shown to exist in multiple DDR genes, including ATM, BRCA2 and CHEK2.

Deleterious (inactivating / loss-of-function) mutation: a mutation involves an alteration in the normal nucleotide sequence of a DNA segment. While most mutations do not confer a selective advantage of the cell carrying it (i.e. passenger/bystander mutation), deleterious mutations result in the protein product of the gene: i) not being produced, ii) getting produced but with abnormal function, or iii) getting produced but interfering with normal cellular function. This should be differentiated from oncogenic (activating/gain-of-function) mutations, which allow cells to proliferate faster. 
DNA damage repair (DDR) / Homologous recombination repair (HRR) genes: DDR genes are the collective name given for large group of genes responsible for encoding proteins that detect and/or repair damage to DNA. The HRR system represents one of the most common DDR pathways specifically responsible for repairing double-stranded DNA breaks. In prostate cancer, key DDR/HRR genes include BRCA1, BRCA2, ATM, CDK12 and CHEK2.

Germline mutation: a mutation that occurs in a sperm cell or an egg cell, and thus is present in all cells of the body and can be passed from parent to offspring. Germline mutations a most commonly detected either utilizing a salivary sample, or a blood sample (specifically white blood cells).

Heterozygous (monoallelic) loss: loss-of-function mutation or deletion of a gene that only affects a single copy (allele) of the gene (assuming a normal diploid genome).

Homozygous (biallelic) loss: loss-of-function mutation(s) and/or deletion(s) of a gene that affects both copies (alleles) of the gene (assuming a normal diploid genome). In malignant tumours, the most common mechanism for homozygous loss involves the combination of a deletion in one allele with an accompanying mutation in the remaining allele. Both events can occur exclusively in the somatic (tumour) genome, or alternatively, may involve an initial inheritance of a germline mutation, followed by a second-hit somatic alteration.

Somatic mutation: a mutation that affects the genome of a cell outside the germ line (e.g. somatic tumour mutation). Mutations of this nature cannot be transmitted to the next generation.

\begin{tabular}{|c|c|c|c|}
\hline \multicolumn{2}{|c|}{ Germline (Richards 2015) } & \multicolumn{2}{|l|}{ Somatic (Li 2017) } \\
\hline Terminology & Definition & Terminology & Definition \\
\hline Pathogenic & $\begin{array}{l}\text { Very strong evidence of } \\
\text { association of gene } \\
\text { alteration with disease } \\
\text { state. }\end{array}$ & $\begin{array}{l}\text { Tier I - variants with } \\
\text { strong clinical } \\
\text { significance }\end{array}$ & $\begin{array}{l}\text { Variants that have } \\
\text { regulatory approval or } \\
\text { endorsed by professional } \\
\text { guidelines to predict } \\
\text { response/resistance to } \\
\text { approved therapies or } \\
\text { have diagnostic and/or } \\
\text { prognostic significance. }\end{array}$ \\
\hline
\end{tabular}




\begin{tabular}{|c|c|c|c|}
\hline $\begin{array}{l}\text { Likely } \\
\text { pathogenic }\end{array}$ & $\begin{array}{l}\text { Moderate to strong } \\
\text { evidence of association of } \\
\text { gene alteration with disease } \\
\text { state. }\end{array}$ & $\begin{array}{l}\text { Tier II - variants with } \\
\text { potential clinical } \\
\text { significance }\end{array}$ & $\begin{array}{l}\text { Variants that have } \\
\text { regulatory approval or } \\
\text { endorsed by professional } \\
\text { guidelines to predict } \\
\text { response/resistance for a } \\
\text { different tumor type, or } \\
\text { investigative therapies } \\
\text { that have some clinical or } \\
\text { pre-clinical evidence. }\end{array}$ \\
\hline $\begin{array}{l}\text { Variant of } \\
\text { unknown } \\
\text { significance }\end{array}$ & $\begin{array}{l}\text { Lack of, or conflicting } \\
\text { evidence to suggest either } \\
\text { pathogenic or benign gene } \\
\text { alteration. }\end{array}$ & $\begin{array}{l}\text { Tier III - variants of } \\
\text { unknown } \\
\text { significance }\end{array}$ & $\begin{array}{l}\text { Variants that have been } \\
\text { reported with unknown } \\
\text { clinical significance, or } \\
\text { variants in cancer genes } \\
\text { that have not been } \\
\text { previously reported. }\end{array}$ \\
\hline Likely benign & $\begin{array}{l}\text { Strong or supporting } \\
\text { evidence to suggest no } \\
\text { association of gene } \\
\text { alteration with disease } \\
\text { state. }\end{array}$ & $\begin{array}{l}\text { Tier IV - benign or } \\
\text { likely benign variants }\end{array}$ & $\begin{array}{l}\text { Variants that are } \\
\text { observed at significant } \\
\text { frequency with no } \\
\text { existing published } \\
\text { evidence of cancer } \\
\text { association. }\end{array}$ \\
\hline Benign & $\begin{array}{l}\text { Strong or very strong } \\
\text { evidence to suggest no } \\
\text { association of gene } \\
\text { alteration with disease } \\
\text { state. }\end{array}$ & & \\
\hline
\end{tabular}

Table 3. Active phase 3 trials of PARP inhibitors in advanced prostate cancer

\begin{tabular}{|l|l|l|l|l|}
\hline Name & $\begin{array}{l}\text { clinicaltrials.gov } \\
\text { identifier }\end{array}$ & $\begin{array}{l}\text { Patient } \\
\text { population }\end{array}$ & $\begin{array}{l}\text { Genomic } \\
\text { selection }\end{array}$ & Intervention \\
\hline AMPLITUDE & NCT04497844 & mCSPC & Yes & $\begin{array}{l}\text { AAP + niraparib vs. AAP + } \\
\text { placebo }\end{array}$ \\
\hline TALAPRO-3 & NCT04821622 & mCSPC & Yes & $\begin{array}{l}\text { Enzalutamide + talazoparib } \\
\text { vs. enzalutamide or placebo }\end{array}$ \\
\hline PROpel & NCT03732820 & $\begin{array}{l}1^{\text {st }} \text {-line } \\
\text { mCRPC }\end{array}$ & No & $\begin{array}{l}\text { AAP + olaparib vs. AAP + } \\
\text { placebo }\end{array}$ \\
\hline
\end{tabular}



Germline and somatic genetic testing in prostate cancer treatment

\begin{tabular}{|c|c|c|c|c|}
\hline CASPAR & NCT04455750 & $\begin{array}{l}1^{\text {st }} \text {-line } \\
\text { mCRPC }\end{array}$ & No & $\begin{array}{l}\text { Enzalutamide + rucaparib } \\
\text { vs. enzalutamide + placebo }\end{array}$ \\
\hline TALAPRO-2 & NCT03395197 & $\begin{array}{l}1^{\text {st }} \text {-line } \\
\text { mCRPC }\end{array}$ & No & $\begin{array}{l}\text { Enzalutamide + talazoparib } \\
\text { vs. enzalutamide + placebo }\end{array}$ \\
\hline MAGNITUDE & NCT03748641 & $\begin{array}{l}1^{\text {st }} \text {-line } \\
\text { mCRPC }\end{array}$ & No & $\begin{array}{l}\text { AAP + niraparib vs. AAP + } \\
\text { placebo }\end{array}$ \\
\hline TRITON3 & NCT02975934 & $\begin{array}{l}2^{\text {nd }} \text {-line } \\
\text { mCRPC }\end{array}$ & Yes & $\begin{array}{l}\text { Rucaparib vs. AAP or } \\
\text { enzalutamide or docetaxel }\end{array}$ \\
\hline KEYLINK-010 & NCT03834519 & $\begin{array}{l}3^{\text {rd }} \text {-line } \\
\text { mCRPC }\end{array}$ & No & $\begin{array}{l}\text { Olaparib + pembrolizumab } \\
\text { vs. AAP or enzalutamide }\end{array}$ \\
\hline
\end{tabular}

AAP: abiraterone acetate and prednisone; mCRPC: metastatic castration-resistant prostate cancer. 